Relations industrielles

Industrial Relations

\title{
Pemartin, Daniel, Gérer par les compétences ou comment réussir autrement?
}

\section{Guy Bellemare}

Volume 55, numéro 2, 2000

URI : https://id.erudit.org/iderudit/051321ar

DOI : https://doi.org/10.7202/051321ar

Aller au sommaire du numéro

\section{Éditeur(s)}

Département des relations industrielles de l'Université Laval

\section{ISSN}

0034-379X (imprimé)

1703-8138 (numérique)

Découvrir la revue

Citer ce compte rendu

Bellemare, G. (2000). Compte rendu de [Pemartin, Daniel, Gérer par les compétences ou comment réussir autrement ?]. Relations industrielles / Industrial Relations, 55(2), 372-375. https://doi.org/10.7202/051321ar

Tous droits réservés (C Département des relations industrielles de l'Université Laval, 2000
Ce document est protégé par la loi sur le droit d'auteur. L’utilisation des services d'Érudit (y compris la reproduction) est assujettie à sa politique d'utilisation que vous pouvez consulter en ligne.

https://apropos.erudit.org/fr/usagers/politique-dutilisation/ 
c'est (aussi) parce que les premiers ont progressivement été articulés et intégrés d'une part par la production de règles spécifiques au réseau, d'autre part par les intermédiaires (matériels notamment) qui lient les acteurs » (p. 244). Dans le prolongement de cette logique de construction de l'action, l'innovation technologique « doit inévitablement accorder une place centrale aux modes de communication et d'échanges d'information entre le système productif et le système institutionnel qui lui est associé » note M. Quere (p. 229) qui s'intéresse dans sa contribution au rôle des pouvoirs publics en tant qu'acteur de premier ordre ainsi que dans l'interaction qu'il peut avoir avec les entreprises et leurs politiques financières en matière d'innovation technologique.

Les différentes contributions qui composent cet ouvrage partagent un souci commun : celui de la pertinence de l'analyse sociétale dans la construction de l'espace social de l'innovation et de la constitution des acteurs qui le composent. Dans cet ouvrage, l'équipe du $L E S T$, fidèle à son habitude, montre à nouveau, avec ce double souci de la rigueur et du respect des points de vue critiques qui la stimulent, que pour comprendre l'entreprise et « ses rapports à la société » on ne peut faire l'économie d'une analyse sociétale qui seule est en mesure de la prendre en considération dans son environnement. C'est-à-dire une analyse qui, dans le cas de l'innovation, en tant que dynamique de transformation, renvoie à une «théorie » de l'entreprise dans la société fondée sur la double interrogation posée par la gestion des rapports de l'entreprise entre son « intérieur » et son « extérieur», d'une part, et, d'autre part, les stratégies de l'entreprise en tant qu'organisation au regard de celles de ses acteurs.

SID-AHMEd SoussI École des hautes études commerciales, Montréal

Gérer par les compétences ou comment réussir autrement

par Daniel Pemartin, Caen : Éditions Management Société, 1999, 126 p., ISBN 2-9126-4734-7.

L'auteur clarifie les raisons et enjeux organisationnels de la gestion par les compétences, en présente les avantages et les inconvénients, identifie les pratiques de gestion des ressources humaines sur lesquelles elle aura une incidence et propose une démarche non réductrice et non modélisante de gestion par les compétences qu'il replace dans une approche systémique.

L'ouvrage s'adresse aux universitaires et aux professionnels intéressés à implanter une gestion par les compétences dans leur entreprise. Celle-ci est présentée comme une solution aux problèmes de motivation du personnel face aux possibilités perçues comme réduites d'opportunités de carrière dans l'entreprise. La gestion par les compétences permettrait aussi d'accroître la flexibilité dans l'utilisation de la main-d'œuvre.
La gestion par les compétences est définie comme une stratégie globale mise en place par la direction d'une entreprise pour clarifier les compétences individuelles et collectives qui lui sont nécessaires aujourd'hui et demain. Elle vise aussi à les définir, les mesurer, les développer et les exploiter, ainsi qu'à repérer des compétences non exploitées. L'auteur est donc un des rares promoteurs à mettre l'accent autant sur les compétences individuelles que collectives. Il note que ce sont les échanges, les interactions dynamiques, les synergies entre les salariés qui autorisent la gestion des aléas, plus que l'initiative individuelle. La complexification des situations tend à relativiser l'importance de l'expertise individuelle au profit de l'expertise collective. Or celle-ci suppose un « vouloir coopérer » et un sentiment 
d'appartenance suffisant au collectif. Cet apport apparaît important car la conception et l'implantation d'un programme de gestion par les compétences seront fort différentes selon qu'on privilégie surtout une optique individuelle ou collective. Par exemple, l'individualisation des salaires, souvent associée à la gestion par les compétences, peut entrer en contradiction avec la notion de compétence collective.

L'ouvrage se divise en cinq chapitres : (1) les enjeux, (2) la notion de compétence, (3) l'organisation qualifiante, (4) la valorisation des compétences et (5) la mise en place de la gestion par les compétences.

Le premier chapitre identifie onze enjeux de la gestion par les compétences, lesquels peuvent être externes (ex. faire face à la montée de la concurrence) ou internes (les conséquences des réductions d'effectifs, la modification des rapports sociaux). Le chapitre deux définit la relation entre les compétences individuelles et collectives et la relation souvent antagonique entre compétence et performance, des aspects rarement explicités dans les autres ouvrages sur la question.

Le chapitre trois analyse l'organisation qualifiante dans laquelle la gestion par les compétences peut mieux s'exprimer. Il explique ses prérequis (débureaucratisation, droit à l'erreur, valorisation de la mobilité et de la flexibilité organisationnelle), son évolution nécessaire (quatre étapes : de l'autocorrection à l'autoamélioration constante) et ses caractéristiques spécifiques. Ces dernières comprennent l'optimisation de l'articulation entre les compétences individuelles existantes, l'intelligence de l'expérience dans une logique de projet, l'enrichissement du travail interprétatif, l'analyse des actions développées par l'expression des controverses ou la résolution des aléas de la production et la reconnaissance des compétences par ajustement des règles de la rémunération. L'organisation qualifiante présente aussi des difficultés. Elle suppose un élargissement considérable de l'espace de liberté des salariés et de leur pouvoir, et la nécessité pour la hiérarchie de ne plus prescrire mais de valider la progression permanente des emplois, ce qui peut être une source d'insécurité pour les cadres.

Le chapitre quatre porte sur la valorisation des compétences. Celle-ci implique le repérage et la capitalisation des compétences exprimées, en reconnaissant ces compétences sur le plan institutionnel au moyen d'un entretien compétences entre l'intéressé et son superviseur, par 1'attribution de responsabilités nouvelles (rémunération subjective), par l'établissement de référentiels des compétences, ou profils de compétences dans la littérature anglosaxonne, pour lesquels l'auteur fait état des difficultés de formulation et d'utilisation et par la rétribution des compétences. La rétribution doit être significative afin de refléter l'importance stratégique que l'organisation affirme leur accorder, sinon, toute la démarche compétences risque d'être un échec par suite de cet effet de double langage. Audelà des niveaux de rémunération, c'est aussi sa structure qui change. Au principe «à emploi égal, salaire égal», se substitue celui-ci : «à compétences exprimées égales, rémunération égale » (p. 92).

L'auteur propose divers modèles de rémunération qui se rapprochent soit de la rémunération de la polyvalence dans le travail industriel ou de la rémunération des niveaux de métier ou d'approches multimétiers chez les employés de métier, des cas classiques dans la gestion par postes. La sélection de ces exemples illustre peut-être la difficulté à trouver des cas de rémunération basés sur une vraie gestion par les compétences. Par ailleurs, l'auteur est un des rares à souligner les ambiguïtés de la gestion par les compétences. Ainsi, le fait de rémunérer la compétence « capacité à faire face au stress " peut servir de prétexte à ne plus considérer le stress 
comme une nuisance psychologique (p. 103). Il propose aussi de prévoir qu'une partie de la rémunération soit basée sur la performance collective au travers de l'intéressement et de la participation, ce qui favorisera l'émergence ou le renforcement des attitudes de coopération.

Le chapitre cinq porte sur la mise en place de la gestion par les compétences. Celle-ci passe par une information large, l'élaboration par la direction de règles précises et simples et par la négociation avec les syndicats de la définition du projet, des référentiels d'évaluation des compétences et d'un cadre budgétaire : «C'est l'acceptabilité d'un projet qui permet la négociation d'un compromis satisfaisant les interêts des uns et des autres, mais c'est le processus de négociation qui rend possible la concertation et la définition des critères d'acceptabilité » (p. 108). Il s'agit là d'une innovation par rapport aux modèles nord-américains d'implantation de la gestion par les compétences qui reposent sur l'exclusion des salariés et de leurs syndicats de la formulation et de l'implantation de la gestion par les compétences. Pour l'auteur, les gestionnaires doivent aussi partager ce projet afin que les salariés puissent disposer des mêmes opportunités de démontrer leurs compétences selon que le supérieur valorisera ou non la délégation. « L'hétérogénéité du management devient alors discriminatoire » (p. 109).

En conclusion, l'auteur considère que la gestion par les compétences n'est pas universelle. Elle s'avère pertinente pour les entreprises qui s'engagent dans une démarche de différenciation, par rapport à la concurrence, basée sur l'innovation (produits ou process), la réactivité au marché, la qualité et apparaît peu compatible avec des stratégies d'entreprises qui visent un avantage concurrentiel par la réduction des coûts ou qui emploient beaucoup de personnel sur des statuts précaires.
Comme tous les promoteurs de la gestion par les compétences, l'auteur néglige certains aspects de la gestion par les compétences que nous relevons ici. L'auteur établit en postulat que la gestion par les compétences est susceptible de motiver des employés et d'accroître la flexibilité dans l'utilisation de la main-d'œuvre. Ce postulat fait bien peu de cas des modalités pratiques de mise en opération de la gestion par les compétences dans les entreprises, lesquelles peuvent mener aussi bien à une démotivation accrue du personnel et à des conflits autour des questions suivantes : quelles compétences seront reconnues? Quelle flexibilité sera mise de l'avant: celle visée par l'auteur, à savoir une utilisation plus flexible du personnel par la direction de l'entreprise ou celle visée par des employés qui voudraient pouvoir davantage gérer eux-mêmes leur carrière dans l'entreprise et à l'extérieur de celleci ? Au-delà, se pose un problème sociétal : comment assurer l'efficacité et éviter une balkanisation du marché du travail si la gestion par les compétences fait l'objet d'aucune normalisation pour fins de reconnaissance externe à l'entreprise?

L'auteur note les limites de la prévision des emplois (postes) mais ne dit pas en quoi la prévision des compétences pourrait être plus assurée, d'autant plus qu'elle doit se fonder sur l'évolution de l'environnement concurrentiel (p. 8), lequel est fortement imprévisible selon Mintzberg (The Rise and Fall of Strategic Management). Pour Pemartin, il y a un déterminisme des compétences puisqu'elles « sont, ou seront, de plus en plus indispensables quelque soit la définition que reçoit le travail » (p. 18).

Le nouveau principe de rémunération « à compétences exprimées égales, rémunération égale » posera d'importants problèmes juridiques en matière d'application des chartes des droits et libertés car les outils actuels d'évaluation des compétences sont loin de présenter le niveau d'objectivité des outils basés sur 
le poste (Lawler, Journal of Organizational Behavior, 1993). Comment s'assurer que la mise en place de la gestion par les compétences ne viendra pas raviver la discrimination systémique en emploi comme l'ont montré Moss et Tilly au sujet des « soft skills » (Work and Occupations, 1996, $\mathrm{n}^{\circ} 3$ ) ?

Ces commentaires critiques, qui s'adressent en fait à la plupart des promoteurs de la gestion par les compé- tences, ne doivent pas faire oublier les qualités de cet ouvrage. Il demeure un de ceux qui vont le plus loin dans l'explication de certains enjeux de la gestion par les compétences et dans la compréhension de la notion de compétence, entre autres son analyse de la relation souvent antagonique entre la performance et la compétence (p. 48-51).

GUY BELLEMARE Université du Québec à Hull

\section{La puissance du stress : une valeur ajoutée}

par Jean-Pierre Hogue et Pierre Brulé, Cap-Rouge et Montréal : Presses Inter Universitaires et Presses HEC, 1997, 167 p., ISBN 2-8944-035-2.

Le stress demeure un phénomène complexe qui touche aussi bien les individus, les organisations que les sociétés. Dans nos sociétés industrielles, la recherche de moyens efficaces pour le contrôler et en tirer avantages a inspiré de très nombreuses publications qui mettent de l'avant les vertus positives du stress comme facteur de croissance personnelle.

La contribution des auteurs Hogue et Brulé se situe dans cette perspective. Le stress est ainsi une énergie qui résulte de l'interaction d'un individu avec son environnement. Tout est énergie, tout est stress, affirment-ils en introduction. Le fil conducteur de l'ouvrage repose sur l'affirmation que le stress est au cœur même de la vie : sans stress, il n'y a pas de vie. Ils se situent dans la même perspective qu'Hans Selye, «le père du stress » qui, dans l'un de ses derniers écrits (Stress sans détresse, 1974), considérait le stress comme un moyen sans lequel il n'est pas possible de s'adapter à son environnement.

Le volume se divise en six chapitres. Les quatre premiers présentent le stress comme une affaire personnelle, une façon d'être et de percevoir son environnement : toute adaptation demeure relative à la perception subjective que l'individu a de son environnement. Selon qu'un environnement est perçu comme peu dangereux, dangereux ou très dangereux, il mobilisera des énergies, des ressources variables à son contrôle, énergies non disponibles pour d'autres fins. Le tout demeure lié à la représentation que chacun se fait du danger, représentation à l'origine de la mobilisation des énergies.

Ainsi, au chapitre I, les auteurs présentent le stress au centre du rythme des changements et des transformations qui bouleversent, déséquilibrent et forcent les gens à l'adaptation constante à leur environnement. Face à ces changements, ils affirment, au chapitre II, que chacun est amené à développer une façon d'être, à partir des énergies générées par le stress, pour vivre et continuer de négocier avec l'environnement.

Le chapitre III présente une comparaison du comportement humain avec le comportement du gestionnaire qui doit transiger dans un environnement en constant changement. Se référant à Mintzberg et à Covey, les auteurs poussent l'analogie en l'appuyant sur la nécessité de gérer l'incertitude l'intégrant à l'émotif, l'intuitif et... au gros bon sens. La démonstration convainc peu en ce qu'elle passe sous silence toutes les données de la recherche dans ce domaine, mais elle fait image. Au chapitre IV, les auteurs associent le stress au phénomène de perception résultant « d'un 